\title{
Evaluando la toxicidad de nanomateriales en modelos celulares tridimensionales ${ }^{\diamond}$
}

\section{Evaluating the toxicity of nanomaterials in three- dimensional cellular models}

\author{
Karla Juárez-Moreno,,,**,» Kathya Angüis Delgado,*** Brenda Palestina Romero,* \\ Rafael Vázquez-Duhalt*
}

\begin{abstract}
Traditionally, in vitro evaluations to determine the cytotoxic effect of nanomaterials in cell cultures have been carried out in two-dimensional cultures, because the protocols to assess it have been adapted from those used in toxicology. However, the interactions between cells are much more complex than those found in a monolayer arrangement. Thus, this is the main reason to promote the implementation of three-dimensional cell cultures also known as spheroids, to assess the effect of nanomaterials on cell cultures. Abundant information supports the idea that spheroids represent a better model for the study of cellular responses, since they emulate more precisely the cellular junctions, communication and physiology that occur in a tissue within an in vivo model. Herein, we discuss some points about the development of 3D cultures as a new and better methodology for nanotoxicological evaluations.
\end{abstract}

KEYWORDS: spheroids, nanotoxicology, cytotoxicity, cell culture.

RESUMEN: Las evaluaciones in vitro para determinar el efecto citotóxico de los nanomateriales en cultivos celulares se han realizado de forma tradicional en cultivos bidimensionales. Esto se debe a que dichos protocolos se han adecuado a partir de aquellos utilizados en la toxicología. Sin embargo, las interacciones entre las células son mucho más complejas que las observadas en un arreglo en monocapa, siendo esta la principal razón por la que, desde hace algunos años, se promueve la implementación de los cultivos celulares tridimensionales, a los que se les conoce como esferoides, para ser usados en las evaluaciones del efecto de los nanomateriales en cultivos celulares. Cada vez son más las evidencias que soportan la idea de que los esferoides representan un mejor modelo para el estudio de las respuestas celulares, pues emulan con mayor precisión las uniones celulares, comunicación y fisiología que sucede en un tejido dentro de un modelo in vivo. En este artículo, discutimos algunos aspectos sobre el desarrollo de los cultivos 3D como una nueva y mejor metodología para las evaluaciones nanotoxicológicas.

PALABRAS CLAVE: esferoides, nanotoxicología, citotoxicidad, cultivo celular.

Recibido: 14 de octubre de 2019.

Aceptado: 17 de febrero de 2020.

${ }^{\diamond}$ Los autores agradecen al proyecto Conacyt INFR-269071, al proyecto de Cátedras Conacyt No. 53 "Nanotoxicología: evaluación toxicológica de los nanomateriales", al proyecto DGAPAPAPIME PE209319, al apoyo brindado por el Departamento de Bionanotecnología del CNyNUNAM y a la Dra. Katrin Quester por su apoyo técnico.

* Universidad Nacional Autónoma de México (UNAM), Centro de Nanociencias y Nanotecnología. Km. 107, Carretera Tijuana-Ensenada, Ensenada, Baja California, México. C.P. 22840.

** Cátedras Conacyt en el Centro de Nanociencias y Nanotecnología-UNAM.

*** Universidad Xochicalco, Escuela de Medicina.

- Autora de correspondencia: kjuarez@cnyn.unam.mx

Correos electrónicos: kathyangu@gmail.com, bpr.roon@gmail.com, rvd@cnyn.unam.mx 


\section{Introducción}

El creciente interés por la evaluación toxicológica de los nanomateriales (NMs) se debe en gran medida a sus diversas aplicaciones, principalmente en las áreas de la salud, alimentación e industrial. La revolución nanotecnológica ha facilitado la síntesis de NMs con propiedades fisicoquímicas peculiares muy interesantes, sin embargo, ha llegado el punto en que la síntesis ha sobrepasado la capacidad de análisis de los NMs. Actualmente las ventajas de las aplicaciones de los NMs son grandes; no obstante su creciente uso en productos comerciales ha despertado un inquietante cuestionamiento sobre su seguridad toxicológica. ¿Será posible que las bondades de los NMs superen sus desventajas? ¿Somos capaces de evaluar el impacto ambiental y de salud que el uso prolongado e ilimitado de los NMs puede ocasionar en los diferentes sistemas biológicos? Y si ese fuera el caso, ¿cuáles son los mejores modelos para su evaluación in vitro e in vivo? En este artículo, exponemos uno de los modelos celulares de reciente uso que se ha adaptado para la evaluación nanotoxicológica: los esferoides. Este modelo celular es utilizado actualmente en las evaluaciones nanotoxicológicas, porque refleja con mayor detalle el comportamiento del arreglo tridimensional de las células cultivadas in vitro, de tal forma que la respuesta celular obtenida sea más parecida a la que podría presentarse en un sistema biológico multicelular in vivo.

\section{¿Qué es la nanotoxicología y cuáles propiedades de los nanomateriales contribuyen a su toxicidad?}

La nanotoxicología es una rama de las nanociencias que surge de forma emergente ante la necesidad actual de evaluar el efecto toxicológico de los NMs en diferentes sistemas biológicos, con la finalidad de explicar los fenómenos y respuestas celulares inducidas por la exposición a los diferentes NMs.

Debido a las peculiares interacciones fisicoquímicas que los NMs exhiben con otros compuestos y moléculas biológicas, ha sido necesario adecuar las técnicas convencionales de la toxicología, para el análisis del efecto de los NMs en diferentes sistemas biológicos. En muchos casos, tales modificaciones no han sido exitosas y son limitadas por las características fisicoquímicas intrínsecas de los NMs como su insolubilidad, capacidad de aglomeración, agregación y sedimentación, entre otras (Xia et al., 2010; Gagner et al., 2012). Además, una característica determinante de algunos NMs es su gran reactividad química con las proteínas y otros componentes presentes en los medios de cultivo celular. Las proteínas presentes en los medios biológicos y que eventualmente pueden rodear al NM forman lo que se conoce como "corona proteica", esta capa de moléculas bioactivas alrededor del NM puede cambiar su química superficial, su tamaño y carga, modulando así sus interacciones con la célula y afectando de forma directa los procesos de internalización del NM y, por lo tanto, la actividad biológica del mismo (Laurent et al., 2012; Zhdanov, 2019). 
Un NM es aquel material que al menos una de sus dimensiones tiene un tamaño entre 1 a $100 \mathrm{~nm}$, existen diferentes tipos, siendo las nanopartículas (NPs) las más estudiadas y utilizadas, estas se caracterizan al estar todas sus dimensiones en la escala nanométrica anteriormente señalada. Existe una enorme cantidad de NMs, con diferentes propiedades fisicoquímicas que afectan sus interacciones y efectos en los sistemas biológicos y, en consecuencia, es muy importante contar con la mayor información relacionada con las características fisicoquímicas de los NMs. Las mediciones típicas iniciales incluyen la determinación de la distribución del tamaño del NM, su forma, composición química, porosidad, química superficial, estructura cristalina y potencial Zeta, estabilidad en medios acuosos, entre otros (Hussain et al., 2015). Si el NM tiene propiedades fisicoquímicas adicionales como, por ejemplo, luminiscencia o magnetismo, entonces se requiere de análisis fisicoquímicos que soporten estas propiedades específicas, por ejemplo, para el caso de los NMs luminiscentes ensayos de fotoluminiscencia, decaimiento y rendimiento cuántico, entre otros estudios que aportan información para sustentar las actividades de estos NM en una célula.

Así pues, para garantizar la seguridad química y biológica de los NMs, es indispensable contar con un estudio completo de sus propiedades fisicoquímicas, para relacionarlas con sus respectivas actividades o propiedades biológicas y las respuestas celulares que puedan ocasionar.

\section{¿Cuáles son los modelos estándares para la evaluación citotóxica de los NMs y qué variables hay que evaluar?}

De forma muy general se considera que un determinado NM puede inducir 4 tipos de respuestas en las células:

1) Cambios en la morfología celular.

2) Cambios en la viabilidad celular (comúnmente conocidos como efectos citotóxicos que involucran la muerte y la proliferación celular).

3) Genotoxicidad (alteraciones o daños en el material genético de la célula), y,

4) Activación de la respuesta inmune (mediante la inducción de una respuesta pro- o anti-inflamatoria en el caso de las células animales, o bien, una respuesta de defensa en el caso de bacterias, protozoarios, hongos y plantas).

Tomando en cuenta estas respuestas, las evaluaciones mínimas dentro de un estudio nanotoxicológico deberían ser los cambios en la morfología celular, citotoxicidad, genotoxicidad e inflamación.

En el caso de los análisis utilizados para determinar el efecto citotóxico en células animales, los modelos estándares para la evaluación in vitro son realizados en cultivos bidimensionales, en los cuales crece un número cono- 
cido de células en una placa o caja de cultivo celular. En este tipo de superficies, las células que tienen la capacidad de adherencia a un sustrato, crecen sobre este formando un estrato continuo de células, a esta estructura se le conoce como "monocapa".

\section{Ensayos para la evaluación de la citotoxicidad de nanomateriales en cultivos in vitro}

Los análisis para medir la viabilidad y proliferación celular en los cultivos bidimensionales en monocapa se realizan, en su mayoría, a través de ensayos colorimétricos. Algunos de estos son muy sencillos, se denominan ensayos de exclusión por colorimetría y se basan en la capacidad que tiene la célula de excluir el paso de un reactivo solo si la membrana plasmática conserva intacta su estructura física, algunos ejemplos de este tipo de reactivos son el azul de tripano o el rojo neutro. En cualquier caso, es posible identificar si las células son viables pues adquieren una coloración diferente a la de las células muertas, por esta razón el nombre del ensayo es exclusión.

Otros análisis colorimétricos más complejos tienen como fundamento medir el cambio de coloración de un reactivo mediante su reducción por enzimas citoplasmáticas o mitocondriales, las cuales tienen un papel fundamental en la fisiología celular. De este modo, el funcionamiento y actividad de estas enzimas, refleja el estado vital y metabólico de la célula. Al reducir el compuesto en cuestión, las enzimas generan un producto con coloración o fluorescencia, que puede ser cuantificado mediante espectrofotometría o fluorimetría y determinar así, con base en la cantidad del compuesto reducido, la cantidad de células existentes. También es posible correlacionar el resultado de estos experimentos con el estado metabólico de las células, después de que fueron expuestas a diferentes concentraciones de NMs y durante una ventana de tiempo definida. Ensayos más elaborados para medir la viabilidad celular, se basan en la cuantificación de moléculas clave del metabolismo celular, por ejemplo, la cuantificación de la concentración intracelular del ATP o de la enzima lactato deshidrogenasa, entre otras.

En este sentido, el fenómeno de la muerte celular pareciera albergar una pregunta determinante: ¿existe la muerte celular inducida por un NM? Sin embargo, la respuesta a esta interrogante no es dicotómica en el sentido de ausencia o presencia. Por el contrario, conocer cuál tipo de muerte celular fue originada por la exposición a un NM, proporciona información particular sobre el efecto y la respuesta celular que un NM determinado puede desencadenar en un sistema biológico.

Por lo tanto, si existe un conocimiento previo de que un NM ocasiona muerte celular, entonces, los análisis posteriores deberían involucrar el estudio sobre el tipo de muerte celular inducida por el NM y determinar si se indujo necrosis, apoptosis, necroptosis o autofagia. La información obtenida en los ensayos permite definir el mecanismo molecular y bioquímico de la to- 
xicidad y deducir a priori si el NM pudiera tener un potencial efecto genotóxico o de inflamación en un determinado sistema biológico.

Si bien los análisis de citotoxicidad han sido establecidos en cultivos en monocapa desde hace muchos años, con el descubrimiento e implementación de los cultivos tridimensionales (3D) se ha generado una controversia sobre si las evaluaciones citotóxicas en cultivos bidimensionales (2D) proporcionan información real sobre la respuesta celular hacia algún agente tóxico (Laurent et al., 2012). Esto se debe a que el crecimiento de una monocapa celular adherida al plástico de la caja de cultivo, provoca que las células adquieran una arquitectura diferente y, por lo tanto, su fisiología y las respuestas celulares ante los estímulos podrían variar (Verjans et al., 2018) y ser diferentes a las observadas en un organismo multicelular, ante un estímulo interno o externo. En este sentido, las respuestas diferenciales entre los cultivos 2D y 3D hacia algunos tipos de fármacos anticancerígenos, sugieren que existen distintas zonas de crecimiento celular muy definidas dentro del esferoide (Jarockyte et al., 2018). Cada una de ellas con características particulares en relación con el metabolismo, disponibilidad de oxígeno, nutrientes y comunicación celular, las cuales están implicadas en la capacidad de un esferoide para responder a la exposición ante un fármaco. Existe un arreglo celular 3D, cuyas interacciones determinan en conjunto la respuesta específica (Kapałczyńska et al., 2018; Huang y Gao, 2018). Además, se ha reportado que los patrones de expresión de proteínas en los cultivos 3D, son más cercanos o similares a aquellos encontrados en los tejidos y en las muestras de tumores primarios (Verjans et al., 2018). De esa forma, desde hace algunos años se han utilizado estructuras celulares tridimensionales para las evaluaciones nanotoxicológicas in vitro, con la finalidad de emular con mayor precisión el comportamiento celular 3D ante un NM determinado, lo cual ha revelado información sorprendente, como se describe a continuación.

\section{Un poco de historia sobre los esferoides}

El arreglo tridimensional de las células animales inicia desde el desarrollo embrionario, esta conformación celular permite el contacto célula-célula y célula-matriz extracelular, fundamental para el mantenimiento de la homeostasis, proliferación y diferenciación celular, entre otras funciones (Laschke y Menger, 2017). Estas estructuras 3D forman naturalmente una esfera multicelular, que se organiza en capas celulares, razón por lo que se les ha denominado esferoides. Este tipo de arreglo celular exhibe diferentes propiedades a aquellas células que crecen en una monocapa 2D, algunas de las características mejoradas por este tipo de estructuras son: incremento en la viabilidad celular, morfología estable, capacidad de polarización, interacciones y uniones celulares, y una actividad metabólica incrementada, (Antoni et al., 2015; Kapałczyńska et al., 2018; Huang y Gao, 2018). 
En los años 50 del siglo pasado se inició la investigación sobre las primeras estructuras celulares in vitro con arreglos 3D para estudios de proliferación e invasión celular (McElwain et al., 1993), pero no fue si no hasta 1971, que existió el primer reporte en donde se utilizó el término "esferoide" para nombrar a este tipo de estructuras celulares en 3D (Sutherland et al., 1971). Ya en los años 90's se empezó a estandarizar la técnica para el cultivo in vitro de esferoides, no solo de células de cáncer sino de otros tipos celulares (Djordjevic y Lange, 1990), siendo así posible reconocer la importancia que tiene la matriz extracelular en la dinámica celular (Nederman et al., 1984).

De esta forma, los esferoides como modelo celular in vitro se utilizaron como una herramienta para estudiar los procesos fundamentales de los mecanismos de la biología celular del cáncer. De acuerdo con los reportes, esto se debe a que el ensamblaje tridimensional de los esferoides es capaz de emular el microambiente celular y, por otro lado, se ha distinguido que las células tumorales y su contraparte in vitro en una conformación tipo esferoide, comparten similitudes no solo estructurales, sino funcionales, moleculares y, por supuesto, biológicas (Weigelt et al., 2015).

Sin embargo, estas estructuras se empezaron ha utilizar en diferentes áreas, por ejemplo, como otro modelo celular en las evaluaciones toxicológicas (Weiswald et al., 2015), para los estudios de farmacocinética y farmacodinamia (Griffith et al., 2006), en la ingeniería de tejidos para el co-cultivo de células (Pampaloni y Stelzer, 2010), para los estudios de migración, crecimiento y diferenciación celular (McElwain y Pettet, 1993), entre otros (figura 1).

Figura 1. Cultivo de células de fibroblastos de ratón L929 para la formación de esferoides.

Cultivo de células para esferoides
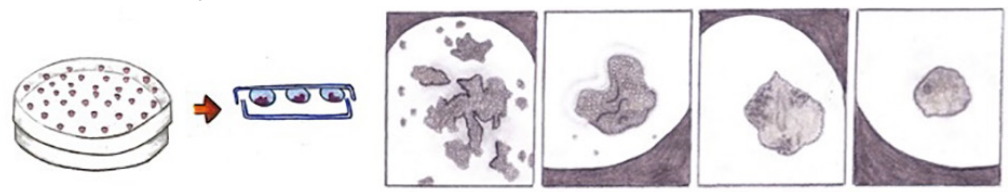

Formación de esferoides de fibroblastos murinos L929 en cultivo
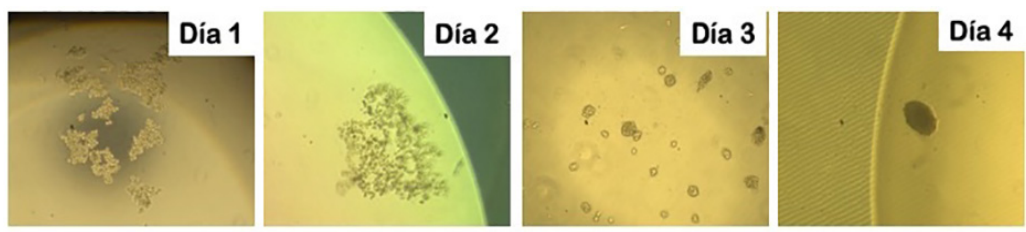

Nota: En la parte superior se ilustra el método de "gota colgante" utilizado para la formación de esferoides y la representación de la formación de esferoides. En la parte inferior, se presentan micrografías de células de fibroblastos de ratón L929, cultivadas para la formación de esferoides, en donde se evidencia el cambio en la distribución de las células conforme aumentan los días del cultivo celular y, finalmente, la formación de un esferoide en el último día de cultivo.

Fuente: Ilustraciones y fotografias proporcionadas por las autoras K. Angüis Delgado y K. Juárez-Moreno, respectivamente. 


\section{¿Cuáles son las ventajas y desventajas de los esferoides respecto de otros cultivos?}

Las ventajas del crecimiento celular en estructuras 3D radica principalmente en que no requieren de un andamio celular para proliferar. Además de ello, se ha observado que el comportamiento 3D de las células arregladas en esferoides se asemeja por mucho al comportamiento que las células tienen in vivo en un organismo multicelular (Fey y Wrzesinski, 2012; Huang y Gao, 2018). En lo que concierne a los estudios de toxicología, se ha reportado que los esferoides son uno de los modelos biológicos preferidos, al tener una mayor vida media y, en consecuencia, ser adecuados para realizar estudios de exposición crónica a ciertos agentes tóxicos (Messner et al., 2013). Además de lo anterior, en el campo de la biología del cáncer, se han utilizado los esferoides porque al igual que en esa patología, las células que los constituyen también son heterogéneas (Huang y Gao, 2018).

Algunas de las ventajas para el uso de los esferoides respecto de su contraparte en 2D, es que permiten el estudio de las interacciones célula-célula y célula-matriz extracelular como sucede en un tumor (tabla 1).

Por otro lado, los estudios realizados en cultivos 3D se han intensificado por la necesidad de generar modelos in vitro de células humanas, lo cual permitirá estudiar a profundidad las respuestas celulares y moleculares derivadas de este tipo de células. Un ejemplo es el caso de la hepatitis $C$, en el que las evaluaciones clínicas de la respuesta a patógenos especie-específicos y la toxicidad hepática ha sido evaluada en cultivos 3D, permitiendo crear un modelo predictivo adecuado, para evitar que las evaluaciones clínicas fallen (Sivaraman et al., 2005).

El cultivo de células en estructuras 3D provee conocimiento sobre la dinámica y mecánica celular, muy diferente al observado en un cultivo 2D. Esto se debe a que las proteínas de adhesión celular y los procesos de señalización pueden ser afectados directamente por el tipo de arreglo celular. Este arreglo tridimensional le permite a la célula depositar la matriz extracelular para comunicarse con el exterior, llevar a cabo procesos de morfo-diferenciación y remodelación, por mencionar algunos procesos biológicos importantes (Griffith y Swartz, 2006).

Se ha demostrado también que los esferoides presentan una mayor resistencia a los fármacos que las células que han crecido en estructuras bidimensionales, este comportamiento es atribuido a las propiedades del microambiente donde se encuentran los esferoides, reflejando con mejor detalle las condiciones existentes en el microambiente tumoral in vivo (Mikhail et al., 2013).

En el caso de las evaluaciones de NMs en sistemas 3D, se ha reportado que la unión e internalización de estos puede ser diferente a la respuesta en monocapa, debido a la compleja fisiología de los cultivos esferoidales. Por ejemplo, en un estudio se realizó la comparación morfológica de 40 líneas celulares de diferentes orígenes tumorales que fueron cultivadas para la forma- 
Tabla 1. Ventajas y desventajas de los cultivos en monocapa (2D) y en esferoides (3D).

\begin{tabular}{|c|c|c|}
\hline & 2D MONOCAPA & 3D ESFEROIDES \\
\hline Ventajas & $\begin{array}{l}\text { Heterogeneidad celular: se } \\
\text { pueden utilizar co-cultivos. } \\
\text { Resistencia a los fármacos. } \\
\text { De fácil manejo y no requiere } \\
\text { matriz especial para su } \\
\text { crecimiento. } \\
\text { Se pueden cultivar células en } \\
\text { suspensión y adherentes. } \\
\text { De bajo costo y alta } \\
\text { reproducibilidad. }\end{array}$ & $\begin{array}{l}\text { Heterogeneidad celular: } \\
\text { distintos tipos de células } \\
\text { pueden ser usadas para } \\
\text { producción de esferoides. } \\
\text { La expresión génica y el } \\
\text { fenotipo son similares a los } \\
\text { tumores in vivo. } \\
\text { Una vez establecidas las } \\
\text { condiciones es de alta } \\
\text { reproducibilidad. }\end{array}$ \\
\hline Desventajas & $\begin{array}{c}\text { No imita la organización 3D de } \\
\text { tumores o tejidos in vivo. } \\
\text { Expresión débil de proteínas } \\
\text { ECM y pobre interacciones entre } \\
\text { células ECM. }\end{array}$ & $\begin{array}{l}\text { Tienen un núcleo necrótico y } \\
\text { una capa periférica de células } \\
\text { con tasa alta de proliferación. } \\
\text { No todas las líneas celulares } \\
\text { pueden formar esferoides y, por } \\
\text { lo tanto, se requiere utilizar una } \\
\text { matriz de inducción del } \\
\text { crecimiento. } \\
\text { Tiempo de crecimiento lento, } \\
\text { además de ser un poco más } \\
\text { costoso. }\end{array}$ \\
\hline Formas de cultivo & $\begin{array}{l}\text { Placas multipozo de cultivo } \\
\text { celular. } \\
\text { Cajas de Petri para cultivo } \\
\text { celular. } \\
\text { Frascos de cultivo. }\end{array}$ & $\begin{array}{c}\text { Placas de baja adherencia. } \\
\text { Placa multipocillo Seivax } \\
\text { NanoCulture. } \\
\text { Placas para cultivo por método } \\
\text { de gota colgante: Perfecta3D } \\
\text { and GravityPLUS 3D. }\end{array}$ \\
\hline
\end{tabular}

Fuente: Elaboración de los autores.

ción de esferoides, con ello se logró identificar tres grupos de esferoides de acuerdo con su arquitectura celular: a) esferoides estrechos; b) esferoides de agregación compacta, y, c) esferoides de agregación laxa (Vinci et al., 2015). Estos modelos in vitro de cultivo celular, tratan de imitar en la medida de lo posible, el microambiente celular in vivo, siendo además de ello, un modelo relativamente sencillo y mucho más barato que los modelos animales (Tchoryk et al., 2019). Por lo que de forma tradicional se ha reconocido a los esferoides como el modelo celular intermediario entre los análisis in vitro y los estudios in vivo (Mikhail et al., 2013). 


\section{Ejemplos de nanomateriales evaluados con esferoides}

La gran mayoría de los estudios reportados sobre el uso de esferoides para la evaluación citotóxica de nanopartículas (NPs) se basan en la comparación de sus efectos citotóxicos, su capacidad de internalización y difusión en cultivos celulares en monocapa (2D) y en esferoides (3D). En ese sentido, se presentan reportes en los cuales se describe la evaluación de NPs de metales y óxidos en estos sistemas tridimensionales.

Se ha evaluado el efecto citotóxico y la capacidad de internalización de nanopartículas de dióxido de titanio $\left(\mathrm{NPs} \mathrm{TiO}_{2}\right)$ en cultivos celulares de osteoblastos humanos para estudiar las respuestas de la interacción célula-célula en el tejido óseo (Souza et al., 2019). Los resultados de esa investigación demostraron que después de 72 horas de exposición a las $\mathrm{NPs}^{\mathrm{TiO}_{2}}$, la viabilidad celular no se afectó. Sin embargo, la incubación de los esferoides de osteoblastos con concentraciones elevadas de $\mathrm{NPs} \mathrm{TiO}_{2}$, influenciaron su ciclo celular permitiendo su diferenciación y mineralización. Un hallazgo importante es que las concentraciones elevadas de $\mathrm{NPs}_{\mathrm{TiO}}$ indujeron la expresión de citosinas pro-inflamatorias y factores de crecimiento involucrados en la homeostasis del hueso y la osteólisis (Souza et al., 2019). En otro estudio, se evaluó la eficacia de la terapia fotodinámica utilizando $\mathrm{NPs}_{\text {de }} \mathrm{TiO}_{2}$ en células de glioma cultivadas en esferoides, los resultados muestran una disminución significativa de la viabilidad celular en las primeras 6 horas post-tratamiento inducida por la radiación con luz UV (Yamaguchi et al., 2010). También se ha evaluado el papel de la deposición de colágeno y la capacidad de diferenciación de células óseas y la producción de citosinas pro-inflamatorias en presencia de $\mathrm{NPs}_{\text {de }} \mathrm{TiO}_{2}$ en modelos 3D de osteoblastos (Souza et al., 2019).

Otras NPs de óxidos que han sido evaluadas en cultivos de 2D y de esferoides son las de óxido de zinc ( $\mathrm{ZnO} \mathrm{NPs}$ ). Un estudio reportó la capacidad de las $\mathrm{ZnO}$ NPs de $25 \mathrm{~nm}$ de diámetro de inducir una respuesta citotóxica de muerte celular dependiendo del tipo de arreglo dimensional que tenían las células del colon (Chia et al., 2015). Una contribución importante de los autores es su propuesta sobre la dinámica de recambio celular presente en los esferoides, los cuales se comportan como "capas de cebolla", cuyas células se desprenden por el daño generado y por los procesos de inflamación causados por la exposición a las NPs; esto permite que exista un capa inferior de células viables la cual responde de forma diferente a los efectos de las NPs dependiendo de su arreglo dimensional (Chia et al., 2015).

Las nanopartículas de oro (AuNPs) también han sido estudiadas en los cultivos esferoidales, y se han reportado NPs ultrapequeñas con tamaños menores a los $10 \mathrm{~nm}$, son capaces de internalizarse con mayor rapidez que las de $15 \mathrm{~nm}$. Además, se determinó que las AuNPs de 2 a $6 \mathrm{~nm}$ se encontraban en el citoplasma y podían penetrar la membrana nuclear, en tanto que las AuNPs de $15 \mathrm{~nm}$ solo se localizaban en el citoplasma. En el artículo publicado, los autores comparan los resultados de los cultivos in vitro, con la 
penetración de las AuNPs en un tumor in vivo y concluyen que los modelos 3D o esferoides representan una buena aproximación para el estudio de la internalización y distribución de las NPs in vitro (Huang et al., 2012). Por otro lado, se han realizado evaluaciones de la internalización de nanopartículas de oro (AuNPs) con diferentes formas como nanoanillos y nanobastones (Yang et al., 2018), en este caso se ha evaluado su papel como nanoacarreadores de fármacos antineoplásicos en cultivos en monocapa y se han comparado con los esferoides (Mikhail et al., 2013). Otros estudios sugieren que la morfología de las AuNPs, ya sean en forma de rodillo o esferoidales, tienen capacidades distintas de poder ser internalizadas por las células (Lu y Stenzel, 2018). Adicionalmente, los esferoides de células de cerebro se han utilizado como modelo in vitro, para determinar la biocompatibilidad y la capacidad de las AuNPs y las NPs de ácido poliláctico de atravesar la barrera hematoencefálica (Matsumoto et al., 2019).

Se ha comparado la toxicidad de NPs bimetálicas de plata y oro $(\mathrm{Ag} / \mathrm{Au}$ NPs), en esferoides de células de cáncer de mama, colon, riñón e hígado, y las mismas células cultivadas en 2D, concluyendo que los cultivos esferoidales son mucho más susceptibles a la toxicidad de las NPs (Katifelis et al., 2018).

Otras NPs no metálicas también han sido evaluadas en los cultivos 3D, por ejemplo, se ha comparado el efecto citotóxico de diferentes concentraciones de NPs de $\mathrm{SiO}_{2}$ de varios tamaños y funcionalizaciones, en cultivos 2D y 3D de células de cáncer de pulmón (A549), carcinoma bucal (células KB) y fibroblastos murinos (L929) (Pellen-Mussi et al., 2018). Los autores encontraron que las $\mathrm{NPs}$ de $\mathrm{SiO}_{2}$ no eran citotóxicas en las células de cáncer de pulmón y los fibroblastos, sin embargo, sí afectaban la viabilidad de las células KB y este efecto era más evidente en los cultivos en esferoides, por lo cual concluyen que la susceptibilidad de las células a las NPs depende del tipo y arreglo dimensional (Pellen-Mussi et al., 2018). También, existen reportes de la incorporación de nanoalambres en esferoides de células cardiacas para generar redes de conductividad eléctrica para mejorar las contracciones de los cardiomiocitos (Tan et al., 2015).

Asimismo, se han realizado estudios sobre la internalización de NPs de poliestireno de 44 y $100 \mathrm{~nm}$ de tamaño en fibroblastos y fibrosarcoma (cáncer de piel) y se encontró que la difusión de las NPs de $100 \mathrm{~nm}$ en los cultivos 3D, era significativamente más lenta que en los cultivos 2D. Es importante mencionar que el citoesqueleto tiene un papel determinante en la internalización de las NPs en los cultivos 3D, no así en los 2D, siendo la macropinocitosis el mecanismo principal por el cual las NPs se internalizan en los cultivos 3D (Belli et al., 2017).

La citotoxicidad de algunos nanomateriales luminiscentes también ha sido evaluada mediante el uso de esferoides, como, por caso, nanopartículas de sílica mesoporosa cargadas con gadolinio (Matsumoto et al., 2019).

Algunos datos muy interesantes relacionados con la comparación citotóxica entre los cultivos 2D y 3D hacia los NMs incluyen algunos estudios que 
reportan que los cultivos esferoidales de células de cáncer de colon (HT29) y células de riñón (SPEV), exhiben una citotoxicidad diferencial a las AuNPs, siendo las de cáncer mucho más susceptibles a la exposición de esas nanopartículas (Pavlovich et al., 2017). Otro caso incluye la susceptibilidad diferencial de fibroblastos (NIH-3T3) y células de cáncer de pulmón (A549) hacia $\mathrm{ZnO}$ NPs y $\mathrm{TiO}_{2}$ NPs. Siendo los esferoides de las células A549 mucho más sensibles a las $\mathrm{ZnO}$ NPs en comparación con los cultivos en monocapa, es probable que esto se deba a la conformación laxa de los esferoides formados por las células de pulmón. Por el contrario, los esferoides formados por los fibroblastos son mucho más compactos, y no muestran una citotoxicidad diferencial hacia las $\mathrm{ZnO}$ NPs respecto de su contraparte en dos dimensiones (Sambale et al., 2015).

Es importante resaltar que las $\mathrm{TiO}_{2} \mathrm{NPs}$ resultaron no ser citotóxicas en ninguna de las dos líneas celulares, sin embargo, el parámetro que se afectó por la presencia de esos NMs fue la formación de los esferoides, ocasionando que su tamaño disminuyera considerablemente (Sambale et al., 2015).

En la actualidad, el uso de cultivos esferoidales para las evaluaciones nanotoxicológicas es una ventaja y es cada vez más evidente que los modelos 3D podrían emular el microambiente celular que existe en un organismo, además al proveer información importante sobre las distintas respuestas asociadas con la arquitectura celular, permite la comparación entre los cultivos bidimensionales en monocapa y el entendimiento de las respuestas celulares hacia los NMs en relación con el arreglo arquitectónico de las células (Lu y Stenzel, 2018).

\section{Conclusiones}

La nanotoxicología es una ciencia emergente originada por la necesidad de evaluar el impacto de los materiales nanoestructurados en la salud y el ambiente, por esta razón, los protocolos convencionales de las evaluaciones toxicológicas han tenido que ser adaptados, y algunas veces su optimización es complicada debido a las características fisicoquímicas de los nanomateriales. En ese sentido, las evaluaciones del efecto citotóxico y de proliferación celular de los nanomateriales se han realizado de forma tradicional en cultivos bidimensionales (2D). No obstante, los cultivos celulares de arreglos tridimensionales se utilizan cada vez más, al existir evidencias contundentes sobre la diferencia en la susceptibilidad que tienen los cultivos 2D respecto de los esferoides o cultivos en 3D.

La mayoría de los estudios apuntan a que los cultivos de esferoides representan una mejor aproximación a la organización de las células en un sistema in vivo, por consiguiente, las repuestas celulares que arrojan emulan con mayor precisión aquellas que se presentan en un modelo in vivo.

La susceptibilidad de las células a los nanomateriales es diferente en los cultivos 2D que en los 3D, y todo indica que las células cultivadas en arreglos 
de dos dimensiones tienen una mayor resistencia a los agentes químicos, necesitando de mayores concentraciones para que estos ejerzan una actividad citotóxica considerable. Con este artículo, evidenciamos la necesidad de implementar los cultivos tridimensionales en esferoides como un parámetro importante de comparación al momento de realizar evaluaciones en materia de nanotoxicología.

\section{Referencias}

Antoni, D., Burckel, H., Josset, E., Noel, G., Antoni, D., Burckel, H., Noel, G. (2015). Three-dimensional cell culture: A breakthrough in vivo. International Journal of Molecular Sciences, 16(12): 5517-5527. https://doi.org/10.3390/ijms16035517

Belli, V., Guarnieri, D., Biondi, M., della Sala, F. y Netti, P. A. (2017). Dynamics of nanoparticle diffusion and uptake in three-dimensional cell cultures. Colloids and Surfaces B: Biointerfaces, 149: 7-15. https://doi.org/10.1016/j.colsurfb.2016.09.046

Chia, S. L., Tay, C. Y., Setyawati, M. I. y Leong, D. T. (2015). Biomimicry 3D gastrointestinal spheroid platform for the assessment of toxicity and inflammatory effects of zinc oxide nanoparticles. Small, 11(6): 702-712.https://doi.org/10.1002/ smll.201401915

Djordjevic, B. y Lange, C. S. (1990). Clonogenicity of mammalian cells in hybrid spheroids: a new assay method. Radiation and Environmental Biophysics, 29(1): 31-46. http://www.ncbi.nlm.nih.gov/pubmed/2305028

Fey, S. J. y Wrzesinski, K. (2012). Determination of drug toxicity using 3D spheroids constructed from an immortal human hepatocyte cell line. Toxicological Sciences, 127(2): 403-411. https://doi.org/10.1093/toxsci/kfs122

Gagner, J. E., Shrivastava, S., Qian, X., Dordick, J. S. y Siegel, R. W. (2012). Engineering nanomaterials for biomedical applications requires understanding the nano-bio interface: A perspective. Journal of Physical Chemistry Letters, 3(21): 3149-3158. https://doi.org/10.1021/jz301253s

Griffith, L. G. y Swartz, M. A. (2006). Capturing complex 3D tissue physiology in vitro. Nature Reviews Molecular Cell Biology, 7(3): 211-224. https://doi. org $/ 10.1038 / \mathrm{nrm} 1858$

Huang, B. W. y Gao, J. Q. (2018,). Application of 3D cultured multicellular spheroid tumor models in tumor-targeted drug delivery system research. Journal of Controlled Release, enero 28; 270: 246-259. Elsevier B.V. https://doi.org/10.1016/j. jconrel.2017.12.005

Huang, K., Ma, H., Liu, J., Huo, S., Kumar, A., Wei, T., ... Liang, X.-J. (2012). Sizedependent localization and penetration of ultrasmall gold nanoparticles in cancer cells, multicellular spheroids, and tumors in vivo. ACS Nano, 6(5): 44834493. https://doi.org/10.1021/nn301282m

Hussain, S. M., Warheit, D. B., Ng, S. P., Comfort, K. K., Grabinski, C. M. y BraydichStolle, L. K. (2015). At the crossroads of nanotoxicology in vitro: Past achievements and current challenges. Toxicological Sciences, 25, septiembre. Oxford University Press. https://doi.org/10.1093/toxsci/kfv106 
Jarockyte, G., Dapkute, D., Karabanovas, V., Daugmaudis, J. V., Ivanauskas, F. y Rotomskis, R. (2018). 3D cellular spheroids as tools for understanding carboxylated quantum dot behavior in tumors. Biochimica et Biophysica Acta - General Subjects, 1862(4): 914-923. https://doi.org/10.1016/j.bbagen.2017.12.014

Kapałczyńska, M., Kolenda, T., Przybyła, W., Zajączkowska, M., Teresiak, A., Filas, V., ... Lamperska, K. (2018). 2D and 3D cell cultures - a comparison of different types of cancer cell cultures. Archives of Medical Science, 14(4): 910-919. https://doi.org/10.5114/aoms.2016.63743

Katifelis, H., Lyberopoulou, A., Mukha, I., Vityuk, N., Grodzyuk, G., Theodoropoulos, G. E., ... Gazouli, M. (2018). Ag/Au bimetallic nanoparticles induce apoptosis in human cancer cell lines via P53, CASPASE-3 and BAX/BCL-2 pathways. Artificial Cells, Nanomedicine and Biotechnology, 46(sup3), S389-S398. https:// doi.org/10.1080/21691401.2018.1495645

Laschke, M. W. y Menger, M. D. (2016). Life is 3D: Boosting spheroid function for tissue engineering. Trends in Biotechnology, 35(2): 133-144. https://doi.org/10.1016/J. TIBTECH.2016.08.004

Laurent, S., Burtea, C., Thirifays, C., Häfeli, U. O. y Mahmoudi, M. (2012). Crucial ignored parameters on nanotoxicology: The importance of toxicity assay modifications and "cell vision." PLoS ONE, 7(1): e29997. https://doi.org/10.1371/ journal.pone.0029997

Lu, H. y Stenzel, M. H. (2018). Multicellular tumor spheroids (MCTS) as a 3D in vitro evaluation tool of nanoparticles. Small, 14(13): 1702858. https://doi.org/10.1002/ smll.201702858

Matsumoto, K., Saitoh, H., Doan, T. L. H., Shiro, A., Nakai, K., Komatsu, A., ... Tamanoi, F. (2019). Destruction of tumor mass by gadolinium-loaded nanoparticles irradiated with monochromatic X-rays: Implications for the Auger therapy. Scientific Reports, 9(1). https://doi.org/10.1038/s41598-019-49978-1

McElwain, D. L. S. y Pettet, G. J. (1993). Cell migration in multicell spheroids: Swimming against the tide. Bulletin of Mathematical Biology, 55(3): 655-674. https:// doi.org/10.1007/BF02460655

Messner, S., Agarkova, I., Moritz, W. y Kelm, J. M. (2013). Multi-cell type human liver microtissues for hepatotoxicity testing. Archives of Toxicology, 87(1): 209213. https://doi.org/10.1007/s00204-012-0968-2

Mikhail, A. S., Eetezadi, S. y Allen, C. (2013). Multicellular tumor spheroids for evaluation of cytotoxicity and tumor growth inhibitory effects of nanomedicines in vitro: a comparison of docetaxel-loaded block copolymer micelles and Taxotere ${ }^{\circledR}$.PloS One, 8(4): e62630.https://doi.org/10.1371/journal.pone.0062630

Nederman, T., Norling, B., Glimelius, B., Carlsson, J. y Brunk, U. (1984). Demonstration of an extracellular matrix in multicellular tumor spheroids. Cancer Research, 44(7): 3090-3097. http://www.ncbi.nlm.nih.gov/pubmed/6373002

Pampaloni, F. y Stelzer, E. (2010). Three-dimensional cell cultures in toxicology. Biotechnology \& Genetic Engineering Reviews, 26: 117-138. http://www.ncbi.nlm. nih.gov/pubmed/21415878

Pavlovich, E., Volkova, N., Yakymchuk, E., Perepelitsyna, O., Sydorenko, M. y Golt- 
sev, A. (2017). In vitro study of influence of Au nanoparticles on HT29 and SPEV cell lines. Nanoscale Research Letters, 12. https://doi.org/10.1186/ s11671-017-2264-9

Pellen-Mussi, P., Tricot-Doleux, S., Neaime, C., Nerambourg, N., Cabello-Hurtado, F., Cordier, S., ... Jeanne, S. (2018). Evaluation of functional $\mathrm{SiO}_{2}$ nanoparticles toxicity by a 3D culture model. Journal of Nanoscience and Nanotechnology, 18(5): 3148-3157. https://doi.org/10.1166/jnn.2018.14619

Sambale, F., Lavrentieva, A., Stahl, F., Blume, C., Stiesch, M., Kasper, C., ... Scheper, T. (2015). Three dimensional spheroid cell culture for nanoparticle safety testing. Journal of Biotechnology, 205, 120-129. https://doi.org/10.1016/j.jbiotec.2015.01.001

Sivaraman, A., Leach, J. K., Townsend, S., Iida, T., Hogan, B. J., Stolz, D. B., ... Griffith, L. G. (2005). A microscale in vitro physiological model of the liver: predictive screens for drug metabolism and enzyme induction. Current Drug Metabolism, 6(6): 569-591. http://www.ncbi.nlm.nih.gov/pubmed/16379670

Souza, W., Piperni, S. G., Laviola, P., Rossi, A. L., Rossi, M. I. D., Archanjo, B. S., ... Ribeiro, A. R. (2019). The two faces of titanium dioxide nanoparticles bio-camouflage in 3D bone spheroids. Scientific Reports, 9(1): 9309. https://doi. org/10.1038/s41598-019-45797-6

Sutherland, R. M., McCredie, J. A. e Inch, W. R. (1971). Growth of multicell spheroids in tissue culture as a model of nodular carcinomas. Journal of the National Cancer Institute, 46(1): 113-120. http://www.ncbi.nlm.nih.gov/pubmed/5101993

Tan, Y., Richards, D., Xu, R., Stewart-Clark, S., Mani, S. K., Borg, T. K., ... Mei, Y. (2015). Silicon nanowire-induced maturation of cardiomyocytes derived from human induced pluripotent stem cells. Nano Letters, 15(5): 2765-2772. https:// doi.org/10.1021/nl502227a

Tchoryk, A., Taresco, V., Argent, R. H., Ashford, M., Gellert, P. R., Stolnik, S., ... Garnett, M. C. (2019). Penetration and uptake of nanoparticles in 3D tumor spheroids. Bioconjugate Chemistry, 30(5): 1371-1384. https://doi.org/10.1021/acs. bioconjchem.9b00136

Verjans, E.-T., Doijen, J., Luyten, W., Landuyt, B. y Schoofs, L. (2018). Three-dimensional cell culture models for anticancer drug screening: Worth the effort? Journal of Cellular Physiology, 233(4): 2993-3003. https://doi.org/10.1002/ jcp.26052

Vinci, M., Box, C. y Eccles, S. A. (2015). Three-dimensional (3D) tumor spheroid invasion assay. Journal of Visualized Experiments, 2015(99). https://doi. org/10.3791/52686

Weigelt, B., Ghajar, C. M. y Bissell, M. J. (2014 ). The need for complex 3D culture models to unravel novel pathways and identify accurate biomarkers in breast cancer. Advanced Drug Delivery Reviews, abril. https://doi.org/10.1016/j. addr.2014.01.001

Weiswald, L.-B., Bellet, D. y Dangles-Marie, V. (2015). Spherical cancer models in tumor biology. Neoplasia, 17(1): 1-15. https://doi.org/10.1016/J.NEO.2014.12.004

Xia, X., Monteiro-riviere, N. A. y Riviere, J. E. (2010). An index for characterization 
of nanomaterials in biological systems. Nature Nanotechnology, 5(agosto): 671675. https://doi.org/10.1038/nnano.2010.164

Yamaguchi, S., Kobayashi, H., Narita, T., Kanehira, K., Sonezaki, S., Kubota, Y., ... Iwasaki, Y. (2010). Novel photodynamic therapy using water-dispersed TiO2polyethylene glycol compound: evaluation of antitumor effect on glioma cells and spheroids in vitro. Photochemistry and Photobiology, 86(4): 964-971. https:// doi.org/10.1111/j.1751-1097.2010.00742.x

Yang, C.-C., Tseng, P.-H., Low, M. C., Hua, W.-H., Yu, J.-H., He, Y., ... Kiang, Y.-W. (2018). Evaluations of cell uptake capabilities of gold nanoparticle and photosensitizer in a cell spheroid, (Conferencia de presentación). En X.-J. Liang, W. J. Parak y M. Osiński (eds.), Colloidal nanoparticles for biomedical applications XIII (vol. 10507: 27). SPIE. https://doi.org/10.1117/12.2287592

Zhdanov, V. P. (2019). Formation of a protein corona around nanoparticles. Current Opinion in Colloid and Interface Science, junio 1. Elsevier Ltd. https://doi. org/10.1016/j.cocis.2018.12.002 